\title{
OPTICAL AND CHEMICAL STUDIES ON SIMULATED INTERSTELLAR GRAIN MATERIALS
}

\author{
J. D. McCULLOUGH, G. R. FLOYD, R. H. PRINCE, and W. W. DULEY \\ Centre for Research in Space Science, and Physics Dept., York University, Toronto, Canada
}

\begin{abstract}
The possibility that many different diffuse interstellar absorption features may be produced by the same type of absorbing atom in different hydrocarbon matrices on interstellar grains has been examined experimentally. The present study shows that absorption bands due to $\mathrm{Na}$ atoms in various hydrocarbon matrices can occur within the wavelength range 5400-5800 \&. A study of the molecules generated from the radiation-induced polymerization of $\mathrm{C}_{2} \mathrm{H}_{2}$ at $55 \mathrm{~K}$ is also reported. It is shown that $\mathrm{C}_{6} \mathrm{H}_{6}$ is an abundant product of this polymerization and may therefore be an important constituent of interstellar grains.
\end{abstract}

The 26 or so diffuse interstellar absorption bands and lines likely provide a definitive clue to the chemical nature of interstellar dust. Laboratory studies which provide simulations of these bands will therefore eventually result in the construction of more plausible models for interstellar grains.

For the past several years we have been examining the spectra of astrophysically abundant metal atoms such as $\mathrm{Ca}, \mathrm{Ti}$, etc., trapped in solid hydrocarbons at temperatures approaching 55 K (Duley, 1968; Duley and Graham, 1969; Graham and Duley, 1971). This model presupposes that some component of interstellar dust consists of pure hydrocarbon or core-hydrocarbon mantle grains. It is this component which is responsible for the occurrence of the diffuse interstellar bands. In this paper we examine the possibility that many different diffuse bands may be produced by the presence of one type of atom in hydrocarbon matrices on interstellar grains. The observation of many diffuse bands then reflects the fact that such grains are composed of a complex mixture of hydrocarbon molecules with relatively few optically active atomic species.

It has been noted previously (Duley, 1969) that $\mathrm{Na}$ atoms trapped in hydrocarbon grains are expected to produce spectral features in the 5000-6000 $\AA$ region due to the shifted Na Doublet at $5890-96 \AA$. Here we examine this proposal in more detail.

Figure 1 shows some spectra obtained recently of Na-hydrocarbon solids at $55 \mathrm{~K}$. These solids are all of the substituted benzene type. We have previously found that such molecules may, because of their chemical stability, be present on interstellar grains (Duley and Graham, 1969; Graham and Duley, 1971). Here the Na atom interacts with one nearby hydrocarbon molecule in the solid to give a single absorption band with peak wavelength between $5600-5500 \AA$ and full width at half maximum intensity of between $\lesssim 20 \AA$ and $100 \AA$. Chemically, the complexes formed in these solids are similar to the well known benzene- $\mathrm{I}_{2}$ complexes. (Andrews and Keefer, 1964; Walkley et al., 1960). Since a weak chemical bond is formed between $\mathrm{Na}$ and a neighbouring hydrocarbon molecule, these complexes are stable thermodynamically 


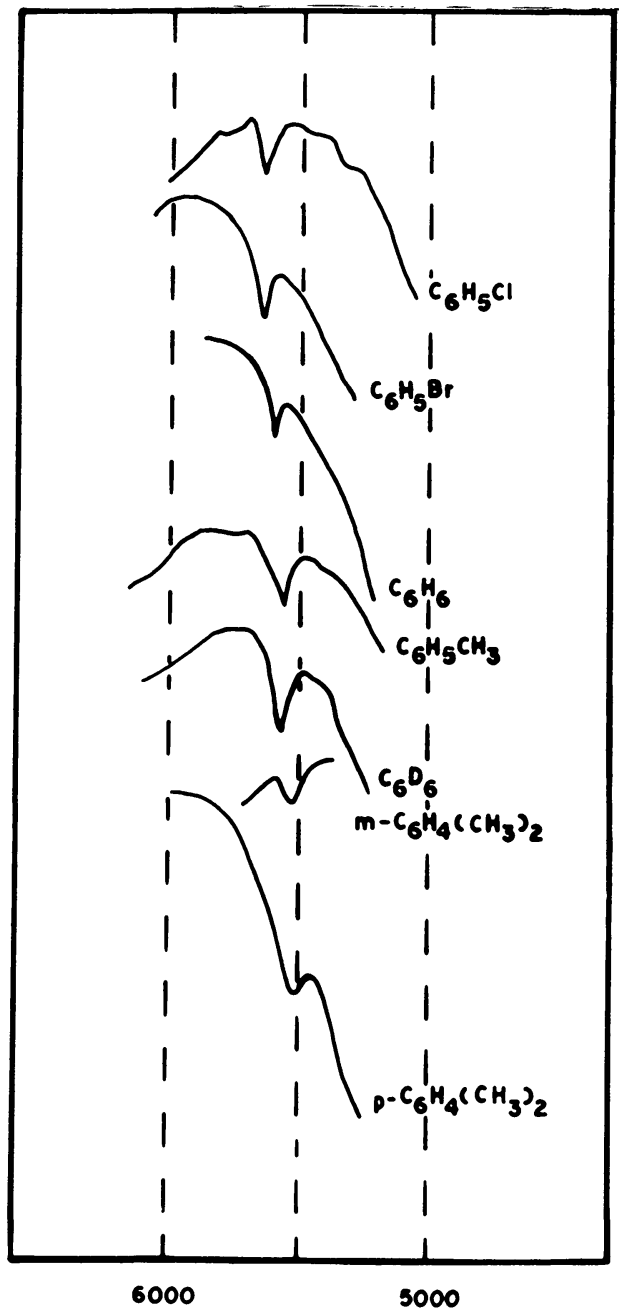

\section{WAVELENGTH (í)}

Fig. 1. Absorption spectra of Na-hydrocarbon solids at $55 \mathrm{~K}$.

to approximately $200 \mathrm{~K}$. In addition, the spectra show no evidence of photobleaching when irradiated with unfiltered light from a tungsten filament.

In Figure 2 we have plotted the peak wavelength of the absorption band in each of these systems against the ionization potential of the hydrocarbon molecule with which the $\mathrm{Na}$ atom is interacting. We see empirically that a simple relationship exists with the characteristic absorption band shifting to longer wavelengths with increasing ionization potential. The molecules complexing with $\mathrm{Na}$ studied to date have a rather narrow range of ionization potentials $(8-9 \mathrm{eV})$. Since other molecules have ionization 


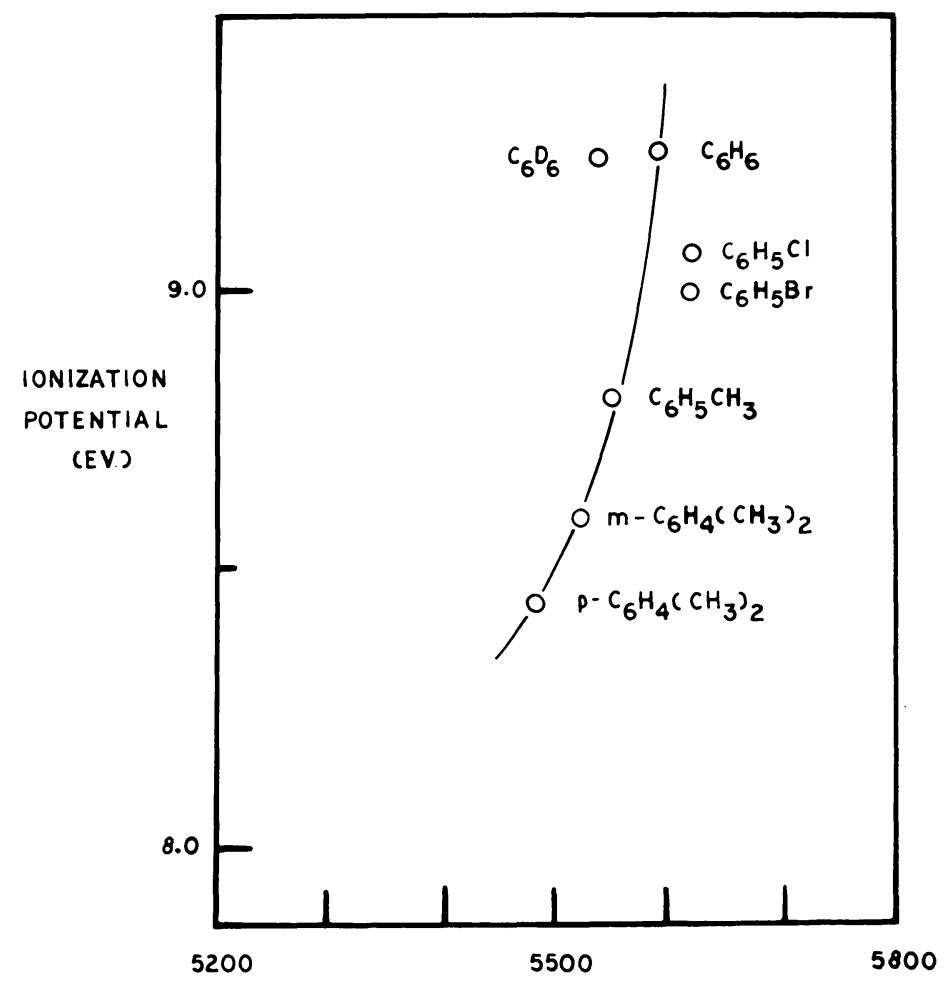

Fig. 2. Wavelength of Na-hydrocarbon band in Figure 1, plotted against the ionization potential of the hydrocarbon molecule.

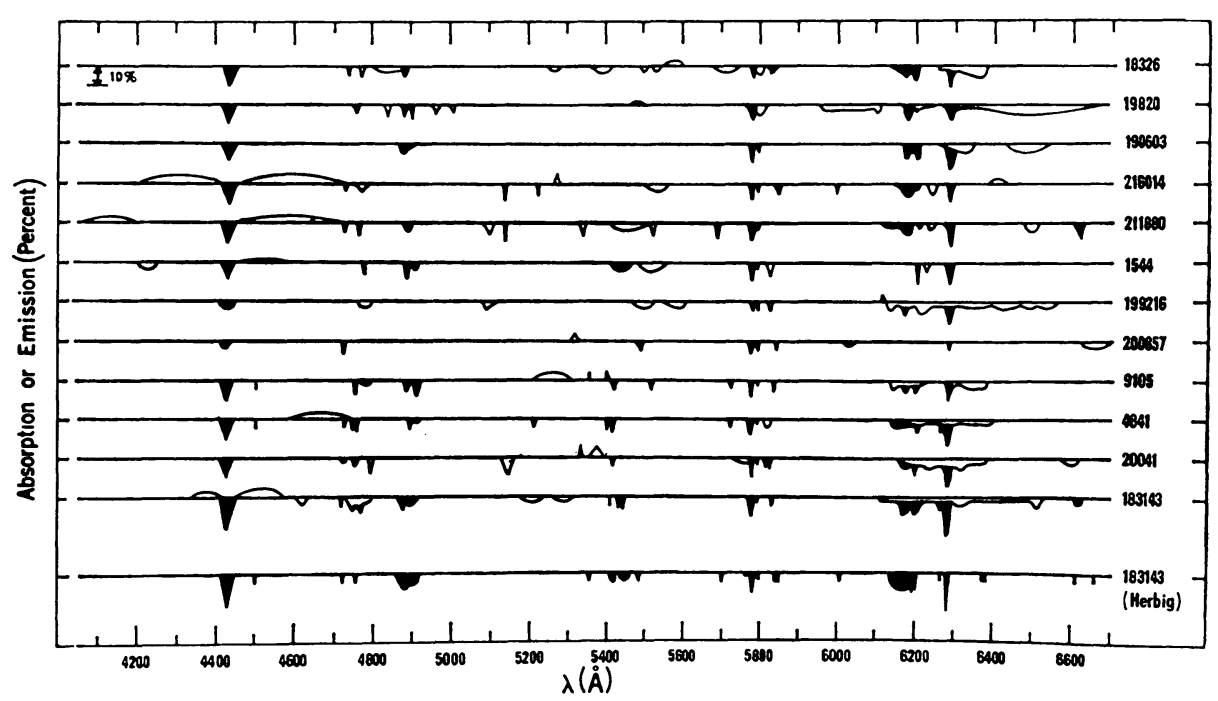

Fig. 3. Diffuse absorption and spectra after York (1970). 
potentials between $\simeq 6.5 \mathrm{eV}$ and $\simeq 13 \mathrm{eV}$ we expect that $\mathrm{Na}$ atoms interacting with other molecules will produce absorption bands in the wavelength range 5800-5400 $\AA$ if this simple correlation is valid. This range contains a number of well known diffuse interstellar bands (Figure 3).

A closer examination of York's (1970) spectra in Figure 3 show that the stars HD 216014, HD 211880, HD 1544 and HD 199216 each have in addition to the usual diffuse bands one or two broad bands between 5600 and $5400 \AA$. Similar features are to be found in the spectra of Walker et al. (1969) and of Honeycutt (1972). The wavelengths of these bands and their width $(\mathrm{FWHM} \simeq 50 \AA$ ) should be compared to those shown for Na-hydrocarbon complexes in Figure 1. If $\mathrm{Na}$ atoms are present in hydrocarbon mantles on interstellar grains then bands would be produced in this region. On the basis of this model the reason that these unusual bands appear at distinctive wavelengths in the spectra of different stars is that different hydrocarbon molecules may predominate on grains in various regions of the interstellar medium. Stationary bands in the spectra of all stars shown in Figure 3 (for example 5780 and $5796 \AA$ ) are due to the presence of certain hydrocarbon molecules on all grains. In this way $\mathrm{Na}$ atoms trapped in mixed hydrocarbon mantles on interstellar grains are able to account for both the stationary and variable wavelength diffuse bands in the 5800-5400 $\AA$ region of the spectrum. It should be noted that the width of the experimental bands has been found to vary from system to system. Thus the variation in width of bands in this region of the interstellar spectrum can be understood.

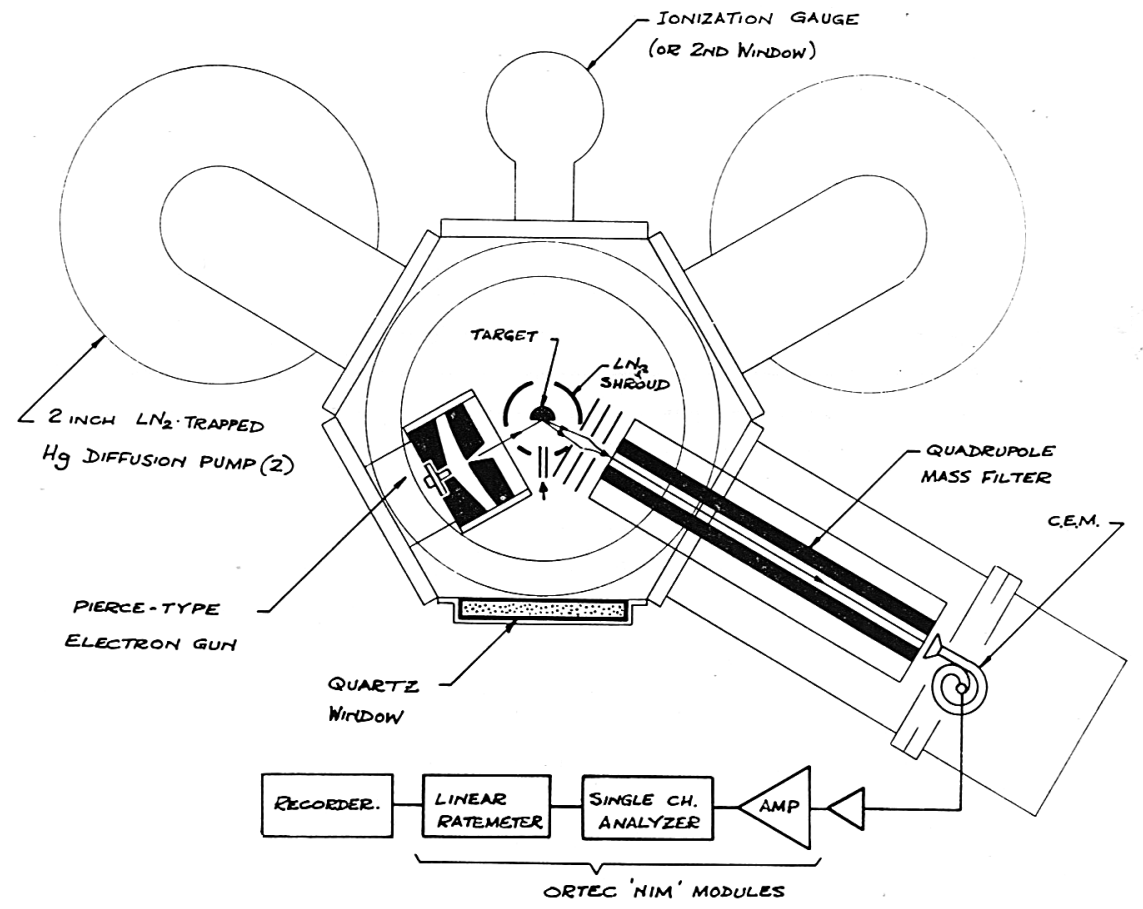

Fig. 4. Schematic diagram of the system used to study products of $\mathrm{C}_{2} \mathrm{H}_{2}$ irradiation at $55 \mathrm{~K}$. 
It is interesting to speculate how molecules as complex as $\mathrm{C}_{6} \mathrm{H}_{6}$ could be produced in the interstellar medium. In this regard we suggest that the simple reaction

$$
3\left(\mathrm{C}_{2} \mathrm{H}_{2}\right)+\text { energy } \rightarrow \mathrm{C}_{6} \mathrm{H}_{6}
$$

may play an important role. This reaction has been shown to occur efficiently in the gas phase (Lind, 1961) with $\gamma, \beta$, $\alpha$ or ultraviolet radiation sources. Realizing that gas phase $\mathrm{C}_{2} \mathrm{H}_{2}$ densities in the interstellar medium are unlikely to be high enough to promote polymerization, we have designed a system which allows us to examine this reaction in solid $\mathrm{C}_{2} \mathrm{H}_{2}$ at $55 \mathrm{~K}$. This system is shown schematically in Figure 4.

$\mathrm{C}_{2} \mathrm{H}_{2}$ is condensed on a finger cooled to $55 \mathrm{~K}$ in high vacuum. The solid is then irradiated with low (10-100 eV) electrons or short wavelength radiation from a $\mathrm{L} \alpha$ source and the ejected molecular ions analysed with a quadrupole mass filter. Prior to an experiment the background gas in the cryostat is mass analyzed by directing electrons from the gun onto the target. Typically background counts in the region

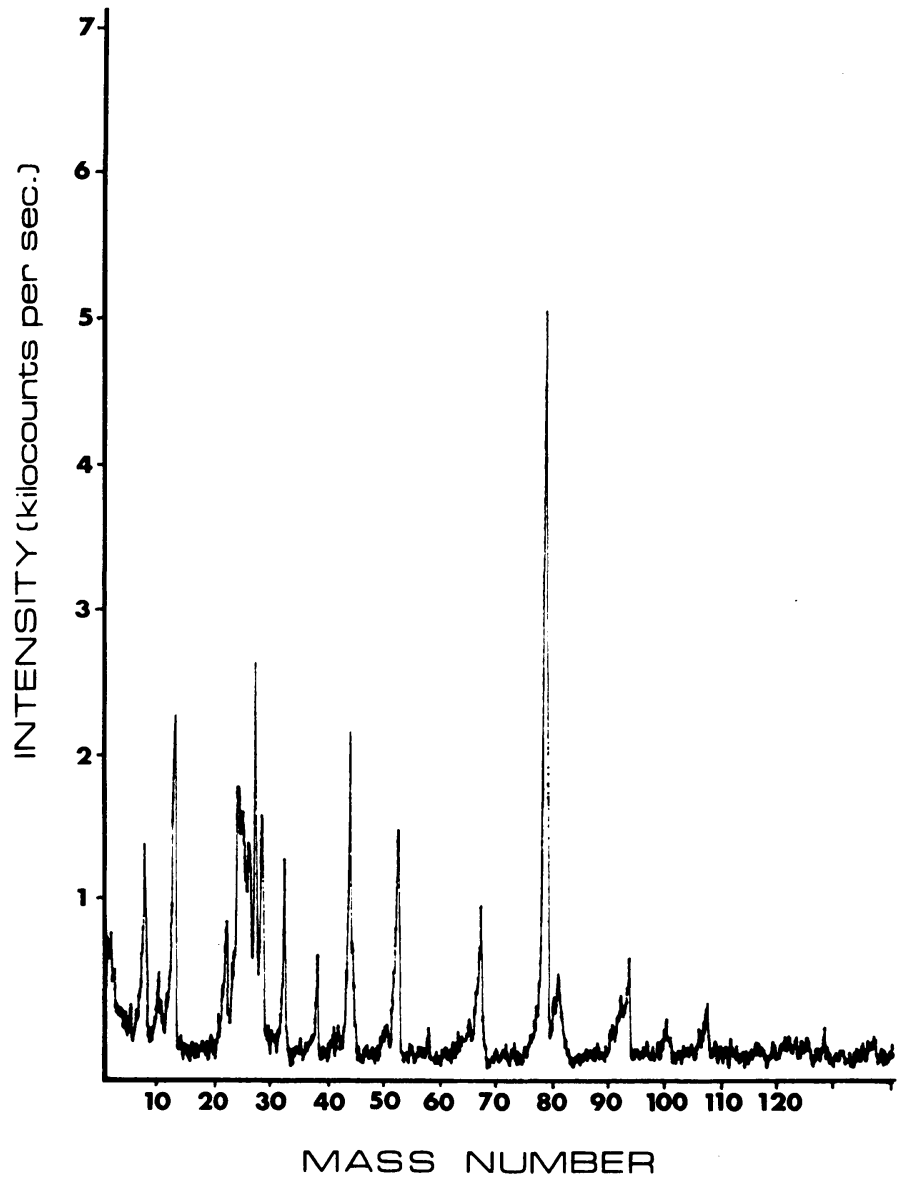

Fig. 5. Mass spectrum of products ejected from solid $\mathrm{C}_{2} \mathrm{H}_{2}$ during irradiation with low energy electrons. 
$m>50$ amu are $\lesssim 40$ counts/sec. This reflects the fact that the system was constructed and assembled without the artificial introduction of any organic solvents, other than methyl alcohol. We feel that a 'clean' system such as this is necessary if experiments on the generation of complex molecules from simple reactants are to provide unambiguous results.

When $\mathrm{C}_{2} \mathrm{H}_{2}$ has been condensed on the target at $55 \mathrm{~K}$, a complex spectrum is obtained as shown in Figure 5. The mass peak at $m / e=78 \mathrm{amu}$ is attributed to $\mathrm{C}_{6} \mathrm{H}_{6}^{+}$. Other peaks at lower masses are characteristic of the cracking pattern of $\mathrm{C}_{6} \mathrm{H}_{6}$. In other experiments to be described more fully elsewhere (Floyd et al., 1972), we have obtained further evidence that the peak at mass 78 does in fact correspond to $\mathrm{C}_{6} \mathrm{H}_{6}$. It should be noted that $\mathrm{C}_{6} \mathrm{H}_{6}$ is far from the only product produced in the polymerization of $\mathrm{C}_{2} \mathrm{H}_{2}$. Figure 5 indicates that the resulting mixture is complex and probably includes members of the alkane, alkene and alkyne series.

Although the existence of $\mathrm{C}_{2} \mathrm{H}_{2}$ in the interstellar medium has yet to be confirmed, much circumstantial evidence for its presence exists. Many of the molecules detected recently in the microwave region (Snyder, 1973) are chemically related to $\mathrm{C}_{2} \mathrm{H}_{2}$. In addition theoretical calculations indicate that $\mathrm{C}_{2} \mathrm{H}_{2}$ may be an important component of some stellar atmospheres (Tsuji, 1964). If these $\mathrm{C}_{2} \mathrm{H}_{2}$ molecules are coated at some stage on the surface if interstellar grains then exposure of these grains to almost any source of radiation will subsequently result in the occurrence of the polymerization reaction given above and in the formation of $\mathrm{C}_{6} \mathrm{H}_{6}$. Obviously this simple reaction is only indicative of the type of reaction that can occur on interstellar grains when they are exposed to interstellar radiation fields. We suggest that $\mathrm{C}_{6} \mathbf{H}_{6}$ may be one of the simpler molecules to be found on interstellar grains. As noted above, hydrocarbon grains containing a mixture of various types of hydrocarbon molecules will, when exposed to a background gas of $\mathrm{Na}, \mathrm{Na}^{+}, \mathrm{Ca}$ and other atomic species, accrete these atoms. The presence of impurity atoms in grains of this sort will give rise to absorption features in the visible region of the spectrum.

\section{Acknowledgements}

This work was supported by National Research Council of Canada grants to W.W.D. and R.H.P.. G.R.F. acknowledges the award of an NRC.C graduate scholarship.

\section{References}

Andrews, L. J. and Keefer, R. M.: 1964, Molecular Complexes in Organic Chemistry, Holden-Day Inc., San Francisco.

Duley, W. W.: 1968, Nature 218, 153.

Duley, W. W.: 1969, Physica 41, 134.

Duley, W. W. and Graham, W. R. M.: 1969, Nature 224, 785.

Floyd, G. R., Prince, R. H., and Duley, W. W.: 1972, unpublished work.

Graham, W. R. M. and Duley, W. W.: 1971, Nature Phys. Sci. 232, 43.

Honeycutt, R. K.: 1972, Astron. J. 77, 24.

Lind, S. C.: 1961, Radiation Chem. of Gases, Reinhold Publ. Corp., New York. 
Snyder, L. E.: 1973, this volume, p. 351.

Tsuji, T.: 1964, Ann. Tokyo Obs. IX, No. 1.

Walker, G. A. H., Hutchings, J. B., and Younger, P. F.: 1969, Astron. J. 144, 921.

Walkley, J., Glew, D. N., and Hildebrand, J. H.: 1960, J. Chem. Phys. 33, 621.

York, D. G.: 1970, Astrophys. J. 166, 65. 